\title{
Adenomiosis apendicular: reporte de dos casos
}

\section{Appendicular adenomiosis: report of two cases}

\section{Ander Morales-Vicente ${ }^{1 *}$, Virginia Ramos-Fernández², Teresa Viñas-Alburquerque³, Norman Gómez-Díaz', Santiago Domingo-Del Pozo y Juana Crespo-Simó \\ ${ }^{1}$ Servicio de Ginecología y Obstetricia, Equipo Médico Crespo; ${ }^{2}$ Servicio de Anatomía Patológica; ${ }^{3}$ Servicio de Ginecología y Obstetricia, Hospital Universitario y Politécnico La Fe; ${ }^{4}$ Servicio de Oncología Ginecológica, Hospital Universitario y Politécnico La Fe; ${ }^{5}$ Equipo Médico Crespo. Valencia, España}

\section{Resumen}

Los adenomiomas son tumoraciones benignas constituidas por un agregado nodular de músculo liso, glándulas endometriales y estroma endometrial. La presencia de adenomiomas fuera del útero es un hallazgo infrecuente. Presentamos dos casos de adenomiomas extrapélvicos localizados en el apéndice. El estudio histológico resultó esencial para el diagnóstico.

Palabras clave: Adenomioma. Apéndice. Histología.

\section{Abstract}

Adenomyomas are a benign tumor compound of smooth muscle nodular aggregate, endometrial glands and endometrial stroma. Adenomyomas presenting outside uterus are a rare finding. Here we report two extrapelvic adenomyomas of the appendix. Histological examination was essential for diagnosis.

Key words: Adenomyoma. Appendix. Histology.

\section{Introducción}

La presencia de estroma y glándulas endometriales en el miometrio se conoce como adenomiosis. Esta patología requiere un estudio histológico para su diagnóstico, describiéndose dos tipos: adenomiosis difusa, cuando afecta de forma generalizada al miometrio, y adenomiosis focal, cuando forma nódulos localizados que se describen como adenomiomas.

La adenomiosis extrauterina puede presentarse clínicamente con dolor abdominopélvico ${ }^{1}$, o ser un hallazgo casual en el transcurso de un estudio por imagen o una intervención abdominal.
El estudio de la adenomiosis uterina suele realizarse mediante ecografía vaginal y abdominal, que muestran masas heterogéneas hipoecoicas correspondientes al tejido glandular endometrial y el músculo circundante', que en el útero generan asimetría de las paredes uterinas y distorsión de la zona de interfase entre miometrio e endometrio²; estas características también se describen de forma similar en el estudio con resonancia magnética ${ }^{3,4}$.

Es rara la presencia de adenomiosis extrapélvica, con muy pocos casos publicados, la mayoría de ellos localizados en el abdomen superior ${ }^{5}$, con frecuencia en el
Disponible en internet: 02-08-2021 Rev Chil Obstet Ginecol. 2021;86(3):317-321

www.rechog.com 0048-766X / @ 2021 Sociedad Chilena de Obstetricia y Ginecología. Publicado por Permanyer. Éste es un artículo open access bajo la licencia CC BY-NC-ND (https://creativecommons.org/licenses/by-nc-nd/4.0/). 
hígado ${ }^{6,7}$, y solo uno descrito en el apéndice ${ }^{8}$. El estudio por imagen inicial se confirmará mediante el diagnóstico definitivo en el estudio histológico posquirúrgico.

La descripción histológica de un adenomioma se corresponde con la presencia de un agregado nodular circunscrito de músculo liso conformado por glándulas endometriales, en general estroma endometrial, que usualmente se localiza en el interior del miometrio".

El presente trabajo expone dos casos clínicos de adenomiosis apendicular confirmados histológicamente tras su exéresis laparoscópica, con el objetivo de dar a conocer esta rara condición clínica, y se repasan su diagnóstico, abordaje y tratamiento. Se trata de dos pacientes que presentaron adenomiosis apendicular confirmada histológicamente tras la resección quirúrgica del apéndice; hasta la fecha solo existe un caso reportado de esta rara localización de adenomiosis ${ }^{8}$.

\section{Caso clínico 1}

Paciente de 34 años en estudio por esterilidad con antecedente quirúrgico de quistectomía laparotómica por endometrioma ovárico derecho en 2008. Diagnosticada ecográficamente y por resonancia magnética de útero arcuato con signos de adenomiosis uterina focal y presencia de hidrosálpinx bilateral. Se realiza salpingectomía bilateral laparoscópica por hidrosálpinx descrito y como hallazgo casual se evidencia un apéndice engrosado de consistencia sólida, por lo que se realizó también apendicectomía.

Se remite la pieza de apendicectomía, que mide $4,3 \mathrm{~cm}$. Tras la sección del apéndice se observa un nódulo en el extremo distal bien delimitado, que mide $1 \mathrm{~cm}$, de consistencia firme y coloración blanquecina (Fig. 1).

El estudio histológico con bajo aumento $(4 \times)$ permite observar la pared muscular apendicular y su luz conteniendo las glándulas propias del apéndice junto con un nódulo, bien delimitado, que se origina en la pared muscular y que presenta luces glandulares en su interior (Figs. 2 y 3). A mayor aumento (Fig. 4) se observa que las luces glandulares, localizadas en el interior de los fascículos musculares, se corresponden con glándulas endometriales acompañadas de estroma endometrial (Fig. 3).

La lesión nodular del apéndice se corresponde con múltiples focos de glándulas endometriales acompañadas de estroma endometrial en el interior de la pared muscular apendicular. Esto provoca una proliferación de células musculares lisas alrededor de esas glándulas

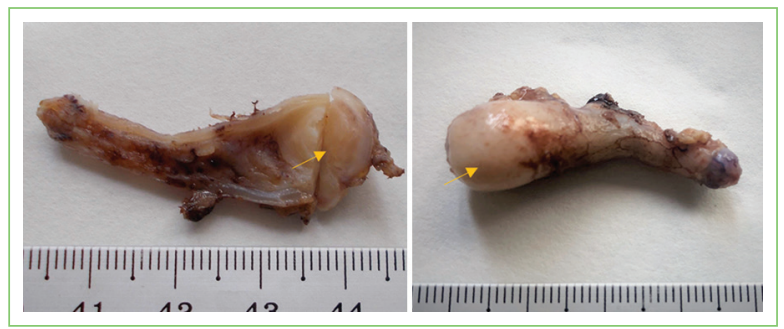

Figura 1. Pieza de apendicectomía con nódulo apendicular.

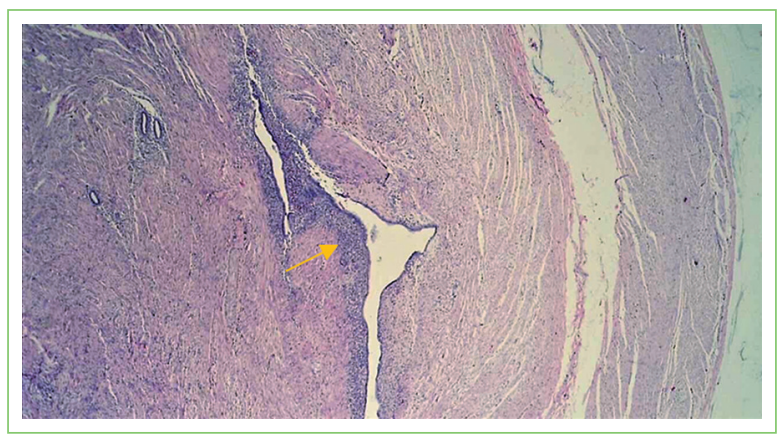

Figura 2. H-E $4 \times$. Lesión nodular bien delimitada originada en la pared muscular del apéndice con luces glandulares en su interior.

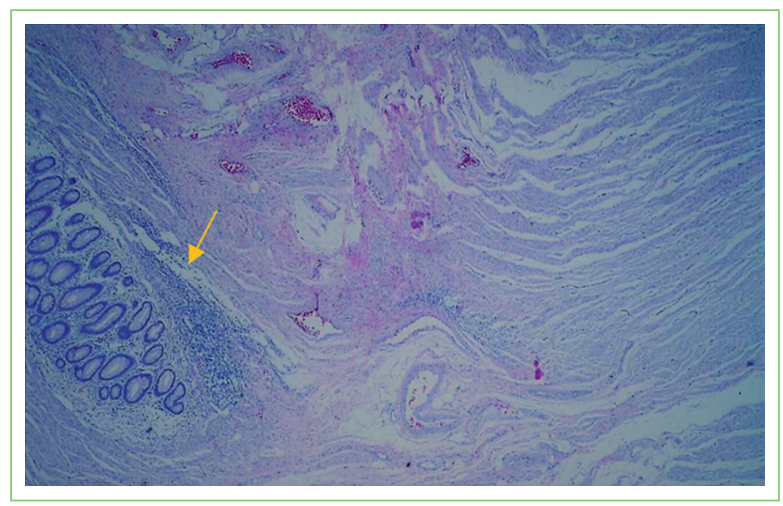

Figura 3. H-E $4 \times$. Mucosa apendicular y pared muscular en la que se observa cómo se origina la lesión nodular a partir de dicha capa muscular.

que constituyen un nódulo bien delimitado en la porción distal apendicular.

La presencia de glándulas endometriales en el apéndice es poco frecuente y la formación de nódulos es muy rara. La presencia de tejido endometrial suele causar una hipertrofia muscular circundante y no es habitual que las fibras musculares formen nódulos tan 
bien definidos. Este hecho nos hace pensar que nos encontramos ante un adenomioma apendicular.

El resto del apéndice estaba revestido por mucosa de arquitectura conservada con folículos linfoides hiperplásicos, que presentaban grandes centros germinales y focos de infiltrados inflamatorios linfohistiocitarios sin extenderse a la pared muscular. No se observaron lesiones histológicas sugestivas de malignidad.

El diagnóstico diferencial histológico de la tumoración apendicular debe hacerse con:

- Adenocarcinoma invasor bien diferenciado: se observan glándulas neoplásicas que no se acompañarían de estroma endometrial y se observarían atipias celulares en el revestimiento epitelial de estas glándulas.

- Endometriosis apendicular: se manifiesta como glándulas endometriales difusas entre las fibras musculares, en la serosa o en la submucosa, sin constituir lesiones focales nodulares bien definidas.

- Leiomioma apendicular: sería similar a la lesión descrita, pero con ausencia de glándulas endometriales.

\section{Caso clínico 2}

Paciente de 45 años, secundípara, con diagnóstico de endometriosis y mala tolerancia al tratamiento con estroprogestágenos. Diagnosticada de endometriosis ovárica bilateral, con dos endometriomas en el ovario derecho $(3,8 \times 2,8 \mathrm{~cm}$ y $3,5 \times 3,7 \mathrm{~cm})$ y uno en el ovario izquierdo $(4,5 \times 3,5 \mathrm{~cm})$.

Clínicamente, la paciente refería goteo intermenstrual y dolor abdominal. Resultaba muy llamativa, en la exploración, la presencia de dolor en la región apendicular. Se planteó tratamiento médico con análogos de la hormona liberadora de gonadotropina durante 6 meses, con buena respuesta. No obstante, la paciente no deseaba continuar con el tratamiento médico y se decidió realizar una quistectomía ovárica bilateral. Durante la intervención se evidenció, de forma casual, un engrosamiento apendicular y se realizó también apendicectomía.

Se remitió la pieza de apendicectomía, que medía 5 $\times 2 \mathrm{~cm}$ y mostraba un área engrosada en su extremo distal de consistencia aumentada (Fig. 5). A la sección se observó una pared de 0,3 cm, sin lesiones. En la zona de la porción distal apendicular se ensanchaba, presentando una tumoración de $1,2 \mathrm{~cm}$ de diámetro, de coloración blanquecina, que dependía de la pared apendicular (Fig. 5).

Histológicamente se observa una mucosa de arquitectura conservada, con folículos linfoides hiperplásicos

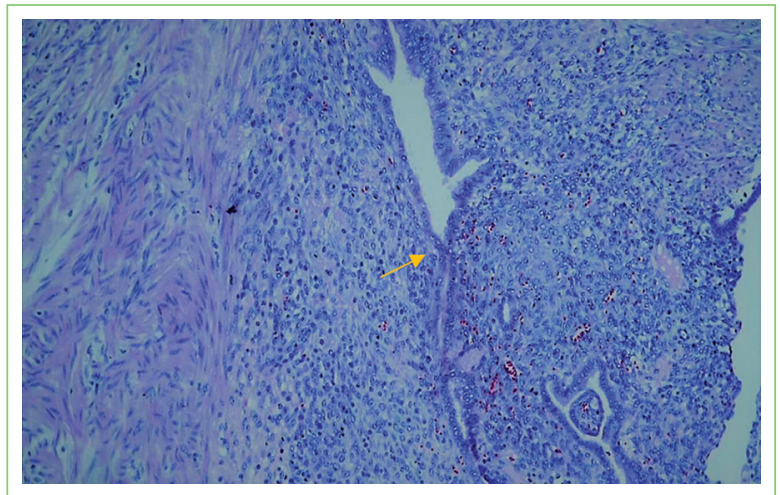

Figura 4. H-E $40 \times$. Presencia de glándulas endometriales que se acompañan de estroma endometrial en el interior de la lesión.

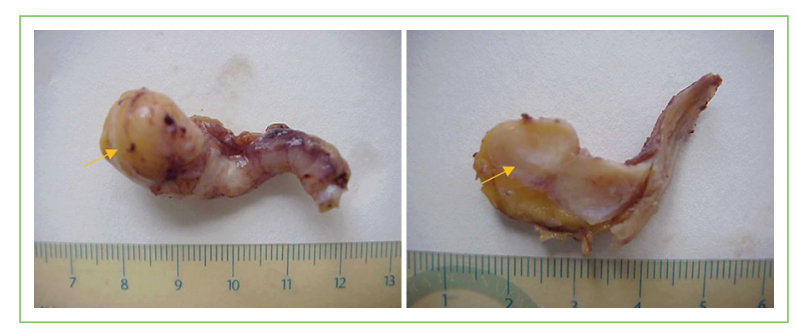

Figura 5. Apéndice con tumoración en su porción distal.

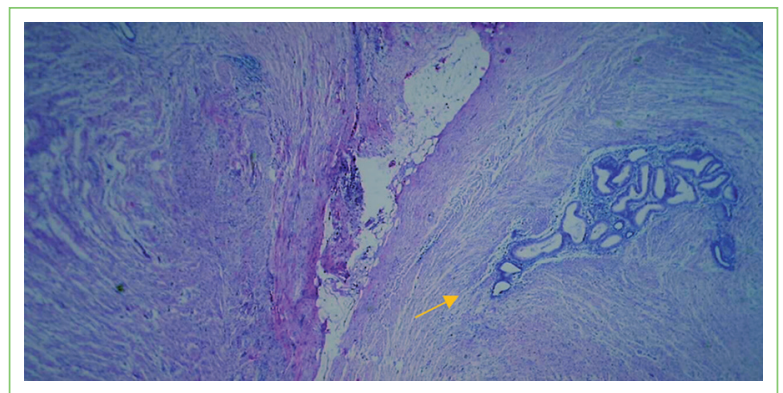

Figura 6. H-E $4 \times$. Pared apendicular con una lesión nodular bien delimitada constituida por tejido muscular liso en cuyo interior se observa un foco de tejido endometrial.

con grandes centros germinales y focos de infiltrados inflamatorios linfohistiocitarios que no se extienden a la pared muscular. La tumoración observada macroscópicamente se corresponde con una hiperplasia muscular de la pared apendicular, en cuyo interior se observan numerosos focos de glándulas endometriales con cambios secretores en su epitelio y rodeadas de estroma endometrial (Figs. 6 y 7). No se observaron lesiones histológicas sugestivas de malignidad. 


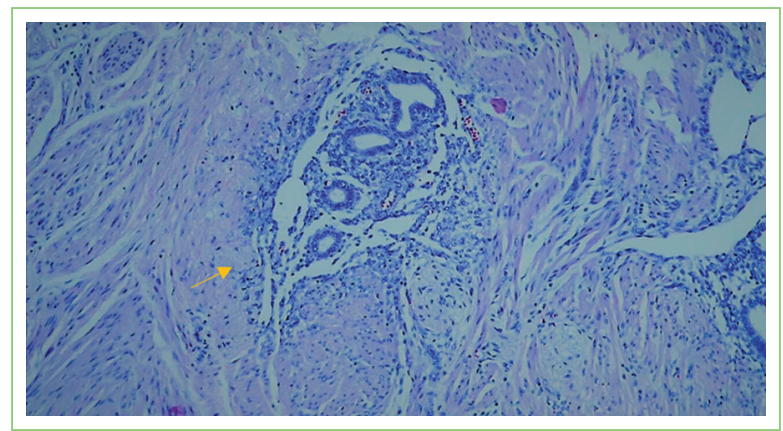

Figura 7. H-E $40 \times$. Glándulas endometriales acompañadas de estroma endometrial con microhemorragias.

También en esta paciente el diagnóstico anatomopatológico de la apendicectomía resultó ser un adenomioma apendicular. La presencia de glándulas endometriales en el apéndice había formado un nódulo bien definido constituido mayoritariamente por fibras musculares.

\section{Discusión}

La adenomiosis extrapélvica es muy infrecuente. Solo existe una decena de casos publicados localizados en el abdomen superior ${ }^{5}$. Se han descrito adenomiomas en el hígado ${ }^{6,7}$ y solo uno en el apéndice ${ }^{8}$.

Los tumores apendiculares son una patología poco frecuente, correspondiendo al $0,4 \%$ de todos los tumores del tracto gastrointestinal. Los más frecuentes son los mucoceles y los cistoadenomas mucinosos o carcinoides, como se ha descrito en algunas series ${ }^{9}$. Encontrar adenomiomas que macroscópicamente simulan tumores primarios apendiculares, como los dos casos descritos, resulta todavía más infrecuente.

En general, el adenomioma apendicular puede cursar de forma asintomática y ser diagnosticado por su hallazgo casual en el transcurso de una intervención abdominal, como ocurrió en nuestro primer caso clínico. No obstante, en otras ocasiones el adenomioma apendicular puede dar lugar a dolor abdominal ${ }^{1}$, como en nuestra segunda paciente.

No podemos concluir que su presencia sea más frecuente en el contexto de una endometriosis grave.

El estudio de la adenomiosis uterina suele realizarse mediante ecografía vaginal y abdominal, que muestran masas heterogéneas hipoecoicas correspondientes al tejido glandular endometrial y el músculo circundante'. El estudio se puede completar con resonancia magnética ${ }^{3,4}$, en la que característicamente la adenomiosis genera asimetría de las paredes uterinas y distorsión de la zona de interfase entre miometrio y endometrio ${ }^{2}$. En el caso de la adenomiosis apendicular es posible identificar también por técnicas de imagen una tumoración nodular 0 un engrosamiento apendicular, sin poder llegar al diagnóstico. El diagnóstico definitivo será histológico ${ }^{1,5}$ tras el estudio de la pieza quirúrgica.

La adenomiosis extrauterina, pese a ser infrecuente, debe contemplarse en el diagnóstico diferencial de las masas abdominales y apendiculares. Un adenomioma se caracteriza como una tumoración benigna en la que el estroma y las glándulas endometriales generan una reacción muscular nodular bien definida y delimitada ${ }^{1,9}$. Debe distinguirse de la leiomiomatosis diseminada, formada por músculo liso. También se diferencia de la endometriosis, ya que esta suele causar hipertrofia muscular circundante, sin ser habitual la formación de nódulos definidos formados por fibras musculares y tejido endometrial, como ocurre en la adenomiosis.

\section{Conclusiones}

La adenomiosis extrauterina es muy infrecuente, pero debe ser considerada en el estudio diferencial de las masas abdominales. Su estudio de sospecha se puede realizar mediante ecografía transvaginal y abdominal, y completarse con resonancia magnética. El diagnóstico definitivo se establecerá posquirúrgicamente con la confirmación histológica por la presencia de glándulas endometriales y estroma endometrial en el seno de una proliferación de células musculares lisas que constituyen un nódulo bien delimitado en la pared apendicular.

\section{Financiación}

Los autores declaran no haber recibido financiación para este estudio.

\section{Conflicto de intereses}

Los autores declaran que no existen conflictos de intereses.

\section{Responsabilidades éticas}

Protección de personas y animales. Los autores declaran que los procedimientos seguidos se conformaron a las normas éticas del comité de 
experimentación humana responsable y de acuerdo con la Asociación Médica Mundial y la Declaración de Helsinki.

Confidencialidad de los datos. Los autores declaran que han seguido los protocolos de su centro de trabajo sobre la publicación de datos de pacientes.

Derecho a la privacidad y consentimiento informado. Los autores han obtenido el consentimiento informado de los pacientes y/o sujetos referidos en el artículo. Este documento obra en poder del autor de correspondencia.

\section{Bibliografía}

1. Paul PG, Gulati G, Shintre H, Mannur S, Paul G, Mehta S. Extrauterine adenomyoma: a review of the literature. Eur J Obstet Gynecol Reprod Biol. 2018;228:130-6.
2. Takeuchi H, Kitade M, Kikuchi I, Kumakiri J, Kuroda K, Jinushi M. Diagnosis, laparoscopic management, and histopathologic findings of juvenile cystic adenomyoma: a review of nine cases. Fertil Steril. 2010;94:862-8.

3. Bazot M, Daraï E. Role of transvaginal sonography and magnetic resonance imaging in the diagnosis of uterine adenomyosis. Fertil Steril. 2018;109: 389-97.

4. Troiano RN, Flynn SD, McCarthy S. Cystic adenomyosis of the uterus: MRI. J Magn Reson Imaging. 1998;8:1198.

5. Sampaio R, García JP, Macedo CS, Vizcaíno JR. A $22^{\text {nd }}$ case report of extrauterine adenomyoma of the abdominal wall. Case Rep Clin Pathol. 2017;4:11.

6. Huanwen W, Hui Z, Xiaowei X, Zhaohui L. Extrauterine adenomyoma of the liver with a focally cellular smooth muscle component occurring in a patient with a history of myomectomy: case report and review of the literature. Diagn Pathol. 2013;8:131.

7. Tandon N, Showalter J, Sultana S, Zhao B, Zhang S. Extrauterine adenomyoma of the liver in a 50 year old female with pelvic endometriosis. Ann Clin Lab Sci. 2017;47:208-12.

8. Kim HC, Yang DM, Kim SW, Kim GY, Choi SI, Park SJ. Uterus-like mass involving the appendix: US and CT findings. J Clin Ultrasound. 2012;40:518-21.

9. Esmer Sánchez DD, Martínez-Ordaz JL, Román-Zepeda PR, Sánchez-Fernández P, Medina González E. Tumores apendiculares. Revision clínico-patológica de 5307 apendicectomias. Cir Cir. 2004; $72: 375-8$ 